\title{
Examinations in paediatric training
}

\author{
Graham S Clayden
}

The examination system in the United Kingdom that is an integral part of the training of paediatricians is often the subject of heated criticism. Whether the emotional energy behind this is fed by prejudices arising from traumatic experiences in their early careers, or by commendable concern for their juniors' unexpected difficulties, is for individual paediatricians to decide. There can be no doubt that there is an enormous expenditure in time and money in both setting and sitting these examinations. To consider this subject dispassionately it is essential to share a clear understanding of the main elements of the discussion. Experience of the United Kingdom examination system for both undergraduates and postgraduates will be used to illustrate the attempts being made to answer some of the questions shown in table 1 .

Table 1 Questions to be discussed when assessing the role of examinations in training

What are we trying to achieve in training in paediatrics?

How important are examinations?

What constitutes a 'good' examination?

How can examiners be helped to conduct useful, fair examinations?

- How can candidates best be helped to pass these examinations?

How can the system be kept flexible enough to: (i) adapt to changing professional requirements, and

(ii) to use advanced educational techniques as they arise?

Training in paediatrics and the examination system in the United Kingdom

Undergraduates are taught paediatrics in university departments as a compulsory part of their medical courses according to the regulations laid down by the General Medical Council. In most universities, however, the final examination in paediatrics is merely a part of the final examination in medicine. After qualification, there is a fork in the path of further paediatric training, one branch leading towards a career as a consultant paediatrician and the other towards a career as a general practitioner with a particular interest in primary care paediatrics. Those aiming for a future consultant post take the two parts of the Membership of the Royal Colleges of Physicians (MRCP). Trainee general practitioners and clinical medical officers may take the Diploma of Child Health (DCH) of the Royal College of Physicians of London or of United Medical Schools of Guy's and St Thomas's Hospitals, St Thomas's Campus, London SE1 7EH

Correspondence to: Dr Clayden.
Health (DCCH) in Edinburgh. Those training in specialties close to paediatrics such as child psychiatry, paediatric surgery, anaesthetics, and paediatric pathology may take either the paediatric option of the second part of the MRCP, or the DCH, to strengthen their claim that they have a special interest in paediatrics.

\section{Aim of training in paediatrics}

The minimum target of training is that every qualified doctor should be aware of the differences between sick children and sick adults. This should reduce delays in diagnosis, reduce the risk of inappropriate treatments, and modify the emotional trauma to the child and family during illness. Every doctor should also be aware of the special needs and vulnerability of children, and know how to help or protect them. These topics should be covered during the undergraduate course, which should also emphasise the association between paediatrics and basic medical science.

\section{Role of examinations in training}

There are two opposing opinions about the role of examinations in training. These can be compared with the 'carrot' and 'stick' methods of activating an obstinate mule. On the 'carrot' side is that the examination is a method of concentrating attention on the subject or task so that candidates may obtain feedback about their performances compared with expected criteria or the performances of peers. This can then be used as a guide to filling in important gaps in knowledge or skills.

The 'stick' side is that the examination is a hurdle to test the motivation of the trainee, a forced compliance with an imposed set of tasks from which a feedback of merely 'satisfactory' or 'unsatisfactory' is given. The gate to the next stage in the career is opened or remains closed, depending on the result of the test. When viewed from these poles, it is clear that examinations that generate feedback must be relevant to the content of the training course, whereas a hurdle can have any content of which the candidate has been warned. In a competitive popular specialty, any hurdle that tests some of the above attributes of the future specialist could be defended as an invigorating example of survival of the fittest.

Many trainees and students will ask for examinations as a target for managing the timing of their studies. Many teachers will depend on the results as a form of evaluation of their methods of teaching and course design. The wider community insists on an objective assess- 
ment of competence to ensure that standards are maintained and that new methods of training are not producing unacceptably different groups of professionals.

Design of examinations and quality control The ideal examination would (1) be highly relevant to the content of the training course; (2) require a method of study or working that develops skills or attitudes necessary for future professional life; (3) encourage the trainers to provide appropriate teaching, time, and facilities; (4) test that the trainee had reached the expected standard of attainment of professional attributes for that particular stage of training; (5) be comparable within and between groups of trainees; (6) be objective, requiring little personal judgment by individual examiners; (7) be conducted by carefully trained examiners whose performance is checked; (8) specify areas of strength or weakness that would guide further training; (9) recognise incompatible attitudes early and match with more appropriate specialties for career guidance; and (10) evaluate the training scheme and suggest modifications. The range of methods of examination includes continuous rating by trainers, tests and tasks during the course, projects to work on, and preparation of a thesis, as well as essays, modified essay questions, multiple choice questions, case commentaries, interpretation of data, recognition of slides, computer assisted interaction, and oral and clinical examinations.

Some of the types of examinations being used at present are given below, and the reader may judge the content of these against the above ideal.

\section{UNDERGRADUATE PAEDIATRIC EXAMINATIONS}

Whether as a formal separate examination or as a part of the final examination in medicine, paediatrics is tested towards the end of the undergraduate clinical course as well as at the end of the paediatric attachment or course. Many departments require their students, individually or in small groups, to complete a project on a subject connected with child health. These entail reviews of published work, the design of questionnaires or surveys, how to deal with patients and parents, analysis of results, and then either written or verbal presentations, or both. Some of the most successful projects have been awarded prizes by the British Paediatric Association and have been received with acclaim by paediatricians. Project work is clearly a forerunner of future professional activity; it teaches teamwork, and methods of retrieving, analysing, and presenting data, as well as generating an active and creative learning approach. It is doubtful whether the students would be able to overcome the inertia of starting a project without the certainty that it will eventually be assessed.

Most people who teach paediatrics to undergraduates grade their students at the end of their attachments. The subjectivity of this process and the dangers of seeming to favour those students who may be known to the teachers through sports, music, or committees, can be reduced by the use of tests during the course. Many departments use multiple choice questions to assess ability to recall facts, oral examinations to test ability to solve problems and some communication skills, and clinical examinations to check on how students deal with patients and skills in examinations. The student's motivation and powers of reasoning and judgment are best evaluated by their paediatric tutor who has provided the opportunity for more detailed discussions. Feedback and advice at the end of the course is often neglected, but may help the students greatly in their future consideration of paediatrics and in their progress through the rest of their undergraduate courses. Their teachers must remember, however, how vulnerable these young students are not only to any sarcasm, but also to distressing clinical experiences. Sometimes poorer than expected performances may indicate that students require help to come to terms with issues arising from their experiences with sick children.

POSTGRADUATE EXAMINATIONS-DIPLOMAS IN CHILD HEALTH

The Diploma of Child Health (DCH) of the Royal College of Physicians (London) is a good example of how it is possible to alter an established examination to accommodate a changing need. Originally the DCH was taken in addition to the MRCP by trainee paediatricians; a large number took the MRCP and only then indicated their special interest in paediatrics by sitting for the DCH. The content of the examination then reflected the orientation of paediatrics in hospitals. The MRCP now has a paediatric option in the second part, and most paediatricians take this. More trainees in general practice, however, who were inspired by their contact with paediatrics during their six months post during vocational training, opt to take the DCH. Most candidates for the DCH of the Royal College of Physicians (London) are trainees in general practice, with a few from community paediatrics, hospital paediatrics, child psychiatry, paediatric surgery, and anaesthetics.

The DCH comprises a preliminary test of 60 multiple choice questions, which excludes the weakest candidates from taking the clinical part of the examination. The content of the multiple choice question test is mainly primary care or preventive child health. A written test has questions that require only short answers on common primary care paediatrics, a question about a case commentary focused on community or chronic paediatrics, and another on more acute child health problems. The questions are set by a panel made up of general practitioners, hospital based paediatricians, community based paediatricians, paediatric surgeons, and child psychiatrists. The clinical part is examined by the same balance of specialties, and includes a long case, during which the candidate takes a detailed history from the parent and child, carries out a full clinical examination, and then presents the findings and discusses management and communication issues with two examiners. 
A different pair of examiners then takes the candidate round a series of children with physical signs. They pay special attention to the candidates approach to the children and technique of physical examination. Developmental testing is also evaluated during this part of the DCH.

A great deal of effort goes into selecting subjects relevant to primary care paediatrics for the multiple choice questions and questions requiring only short answers. Each question is tested for its discriminating power (that is, how well do candidates score on the individual questions correlated with their scores on the whole test). Questions that discriminate poorly can then be modified, usually by removing any ambiguity of the language or making the question slightly easier. Although individual candidates who fail are offered advice, there is no detailed feedback of poorly answered questions, or reports to the candidates of weak or misunderstood areas. With the increasing use of modern technology and increasing 'glasnost' of examining boards, more detailed feedback may be forthcoming.

An analysis of the behaviour of the various types of professional examiners in the DCH examination showed that they were of similar generosity or meanness in their awarding of marks. When the written papers were marked by two examiners there was a clear similarity in the marks given.

The DCH of the Royal College of Physicians (Glasgow) also comprises a written paper on the principles of child health with particular reference to community child health, and clinical and oral examinations.

The Diploma of Community Child Health (DCCH) is jointly managed by the Royal College of Physicians (Edinburgh), the Royal College of General Practitioners (RCGP), and the Faculty of Community Medicine (Public Health) of the Royal Colleges of Physicians of the United Kingdom. ${ }^{1}$ The DCCH comprises an assessment of written case reports submitted by the candidate, a written paper with a modified essay question with six to 10 sequential questions, and 10 to 20 questions requiring short answers. There is an oral examination but there was no clinical examination until the 'objectively structured clinical examination' (OSCE) was introduced. Some clinical skills are assessed with written questions about material on still photographs or video sequences.

An important question to ask is whether the DCH or DCCH are driving the studies and the demand for experience of the trainees in an appropriate direction. Clearly the emphasis on primary care is valuable and by including developmental, vision, and hearing testing in the clinical examination it has encouraged the trainees to become familiar with these essentials of developmental surveillance. There are a number of general practitioners and paediatricians, however, who feel that these diplomas give inadequate confirmation that a trainee general practitioner has reached a sufficiently high level of competence to carry out child health surveillance.

POSTGRADUATE EXAMINATIONS-MRCP (PART I) The MRCP (Part I) is a paper containing 60 multiple choice questions that aims to test a broad knowledge of medicine. It includes questions about basic science, and also about particular diseases, and includes psychiatry and paediatrics. The questions are set by an examining board that has representatives from the three Royal Colleges of Physicians of the United Kingdom, including at least one paediatrician, a psychiatrist, and consultants in general medicine. The balance of the questions is decided by the examining board, and the discriminating power for these multiple choice questions is also analysed in the same way as described for the DCH. It is acknowledged that multiple choice questions inevitably test recall of facts, and only rarely touch on slightly higher levels of reasoning and judgment. They also have the disadvantage of giving a clue to the answer but the candidates do have to make a clear decision between 'true' or 'false' or accept that they do not know. They are penalised with a negative mark if they guess unsuccessfully. It could be strongly argued that this penalty for not honestly admitting one's ignorance is an appropriate feature of an examination that is an entry test for consultant training.

Paediatricians seem to feel more unhappy about the MRCP (Part I) examination than about any other educational issue. There is a fear that it is unfair to those who intend to become paediatricians, although they acknowledge that it is usually taken by senior house officers (SHOs) before they have travelled far into their specialist paediatric training. The main anxiety is that if it is competitive, the paediatric SHO who is first on call and frequently deprived of sleep (especially in neonatalogy) is at a disavantage compared with the medical SHO who is shielded by preregistration house officers. There is also concern that the examination might pose difficult questions about diseases associated with psychogeriatrics or other specialties with which the paediatric trainee is inevitably out of touch. A study was therefore carried out by the MRCP (Part I) Board to look into some of these anxieties and to consider whether any changes were indicated.

A previous study carried out between 1972 and 1976 showed that those candidates who eventually took the paediatric option in Part II had a similar rate of passing Part I at their first attempt as those later opting for Part II in general medicine. ${ }^{2}$ Those who eventually took the paediatric Part II, however, had taken significantly longer than those who took the Part II in general medicine (average 34 months from qualification compared with 27 months, respectively).

In the recent study the results at Part I for the 290 entries to the paediatric option at Part II and the results for the 1490 general medical entries during the year 1989 among graduates from medical schools in the United Kingdom were compared. Again the future paediatricians had taken longer to obtain their Part I passes than those taking general medicine (average 37 months compared with 27 months, respectively, in 1989). When this was analysed further, it appeared that it was partly because more paediatricians required more attempts to pass 
Table 2 Entrants for the paediatric option for the second part of the MRCP in 1989 compared with those who took the general medical option for the number of times they took the first part before they were successful

\begin{tabular}{lll}
\hline $\begin{array}{l}\text { No of attempts } \\
\text { at Part I } \\
\text { before passing }\end{array}$ & $\begin{array}{l}\text { No (\%) who took } \\
\text { the paediatric } \\
\text { option }\end{array}$ & $\begin{array}{l}\text { No(\%) who took } \\
\text { the general } \\
\text { medical option }\end{array}$ \\
\hline 1 & $103(36)$ & $767(52)$ \\
2 & $96(33)$ & $438(29)$ \\
3 & $55(19)$ & $195(13)$ \\
4 & $36(12)$ & $90(6)$ \\
\hline Total & $269(100)$ & $1490(100)$ \\
\hline
\end{tabular}

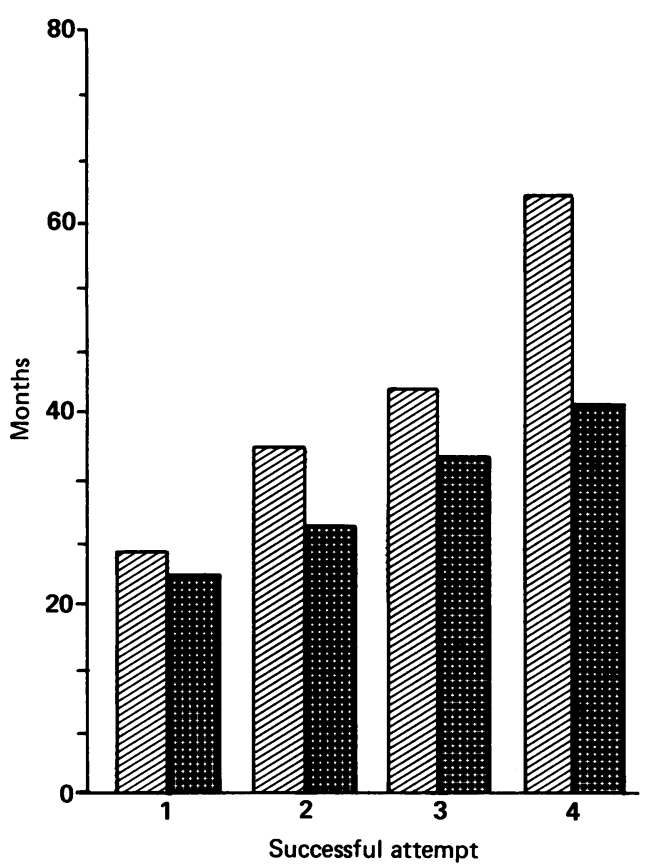

Interval between qualification and success in the first part of the MRCP examination. The striped bars represent those who took the paediatric Part II, and the cross hatched bars those who took the Part II in general medicine.

Part I (table 2), and partly because paediatricians waited longer to make these attempts if they were unsuccessful on the first attempt (figure).

Table 2 shows that the paediatricians took significantly more attempts to pass Part I than the general medical candidates. When all the Part I scores for all attempts for the 290 paediatric entries and the 1490 general medical entries were considered, the mean was 58.22 for paediatricians and 60.48 for those doing general medicine.

The delay in making further attempts to pass Part I can only further disadvantage paediatricians, so they should be given all the help they can to take Part I as soon as possible.

As part of the study for the MRCP (Part I) Board, this small but real disadvantage was investigated by a paediatrician, who analysed the content of the items of nine Part I papers between 1983 and 1990. Items were judged according to their advantage or otherwise to paediatricians (table 3 ).

The report of this study has recently been discussed in depth by the Royal Colleges of Physicians of the United Kingdom and the
Table 3 Analysis of the content of 2700 multiple choice questions in the MRCP first paper for potential advantage or disadvantage to trainee paediatricians

\begin{tabular}{lc}
\hline Degree of advantage & Percentage of items \\
\hline Clear paediatric disadvantage & 15 \\
Some paediatric disadvantage & 9 \\
No disadvantage or advantage & 62 \\
Some paediatric advantage & 5 \\
Clear paediatric advantage & 9 \\
\hline
\end{tabular}

recommendation of the Part I Board to reduce this small imbalance has been endorsed. It is planned to increase the number of 'no disadvantage' questions from $62 \%$, and to balance the 'clear advantage' questions as much as possible in future. A shortage of multiple choice questions on important paediatric topics that would be fair to ask the general medical candidates is slowing down this process, and paediatricians are invited to supply material either to the British Paediatric Association or directly to the Royal Colleges of Physicians.

POSTGRADUATE EXAMINATIONS-MRCP (PART II) Trainee paediatricians have an option when they have succeeded in MRCP (Part I) to take the Part II in paediatrics. The examination consists of a written paper, which is made up of questions about interpreting data, case commentaries, and projected material. This written paper achieves a fair degree of objectivity as marking keys are given to the examiners who meet in groups to score, which allows for unexpected responses from candidates to be discussed and awarded a reasoned mark.

To increase the value of the MRCP (Part II), paediatricians are invited to supply case histories, results of investigations, and clinical photographs of children by whose cases they felt that they had been tested, whether they were successful or not. This would aim the questions at subjects that are testing for practising paediatricians.

Writing and editing questions based on this material is time consuming and rather daunting the first time. Paediatricians are requested to send even raw case material to the Royal Colleges of Physicians or the British Paediatric Association for processing into questions of the usual type (and more innovative questions for the future) where they will be edited and then sent on to the examination boards and question groups of the Royal Colleges of Physicians. When their questions are used, members will be notified about how the candidates did, and how discriminating their questions were.

Sharing the responsibility for evaluating training and trainees

Supplying clinical material and questions to examination boards, agreeing to become examiners, helping to host clinical examinations in hospitals, and practising being examiners with junior staff and students, will all help in maintaining a fresh and relevant examination system. The Royal Colleges, but perhaps less so the universities, take care to pair an experienced 
examiner with a less experienced one. Most examination boards keep a check on any extremes of marking by examiners by feeding back the examiner's place on a 'hawk-dove' scale. More junior paediatricians should be trained to examine, and this is equally true for other essential techniques in medical education. Little encouragement is likely to be given to junior academics to develop these skills, however, if they compete with time for research.

\section{Helping trainees to pass examinations}

Helping the trainee to pass one of these examinations is an obligation on the trainer; paediatricians are beginning to recognise what general practitioners have known and practised for sometime. The junior paediatric grades are training grades and time should be allocated to teach, appraise, and coach. This is a fair trade off for the reduced workload of consultants fortunate enough to have adequate junior staff, and is an extremely useful way of keeping up to date. This effect of the system is often forgotten in the discussions about whether examinations are necessary or desirable.

Another important contribution to training and continuous medical education is the production of teaching material and review articles in journals. It has always surprised me that so little time is allocated in annual meetings of the main scientific associations to teaching sessions, which would provide valuable 'updates' for practising paediatricians who would return to their trainees armed with the most modern understanding of the management of important clinical problems.

Maintaining flexibility of examination system Fortunately the universities and Royal Colleges are aware of the changing nature of medical practice and the role of the doctor in health management. What is less evident is the response to new techniques. Few examinations include videos of important physical signs, which might be too transient or too distressing to have as short cases. Interactive management of problem cases could be constructed by combining the existing parts of the written examinations of the DCH or MRCP, but the fear of computers is collaborating with the normal inertia of accepting new techniques.

\section{Conclusion}

Examinations are necessary for feedback to trainees and to ensure a standard of proficiency, especially in dealing with ill children. The DCH examiners are exorted to reject doctors who they would not want to treat their own children. We should all share the responsibility of making the examination system work in favour of children by providing them with well trained and tested doctors who have not wasted their time learning unnecessary trivia.

I thank Dr Peter Fleming, Chairman of the Part I Board, for encouragement, Dr Alastair Vale, Secretary to the Part I Board for checking the Part I statistics, the examinations and computer departments of the Royal College of Physicians for finding the data, to the Presidents, Censors, and Officers of the Royal data, to the Presidents, Censors, and Officers of the Royal
Colleges of Physicians in the United Kingdom for their permission to publish the results, Dr Bill Uttley for information on the Edinburgh Diploma of Community Child Health, and the Board help in the various studies of examiners' performance.

1 Roberts GD, The diploma in community child health. Horizons 1989:538-42.

2 Clayden GS, Paediatric option in MRCP examination. Med Educ 1977;11:119-24. 